\title{
MARATHON ADAPTATION TO SPATIAL CCNTRAST: SATURATION IN SIGHT
}

\author{
Svein Magnussen* and Mark W. Greenlee \\ Neurologische Universitätsklinik mit Abteilung für Neurophysiologie, Hansastr. 9 , \\ D-7800 Freiburg im Breisgau, F.R.G
}

(Received 24 January 1985; in revised form 6 May 1985)

\begin{abstract}
The contrast thresholds for detecting a $6.0 \mathrm{c} / \mathrm{deg}$ vertical sinusoidal test grating were tracked during and after $3 \mathrm{hr}$ inspection of a high-contrast adapting grating of the same spatial frequency and orientation. Log contrast threshold increased linearly with log adaptation time, attaining a final stable value after approximately 30 and $60 \mathrm{~min}$ of adaptation for the two subjects tested. The recovery function was likewise linear on double logarithmic axes. The results further suggest that adaptation beyond the saturation point had no influence on the subsequent rate of recovery.
\end{abstract}

Grating adaptation Saturation Psychophysics

\section{INTRODUCTION}

Prolonged inspection of high-contrast luminance gratings increases the contrast threshold for detecting subsequently presented test gratings of similar spatial frequencies and orientations, decreases the perceived contrast of suprathreshold gratings, and shifts the perceived orientation and spatial frequency of test gratings whose physical orientation and spatial frequency differ somewhat from that of the adapting grating (Braddick et al., 1978). Earlier studies of the time course of these spatial aftereffects suggested that they reached a maximum value already after 1-3 min of adaptation (Gibson and Radner, 1937; Blakemore and Campbell, 1969; Blakemore et al., 1970, 1973). A further increase in adaptation time might, however, cause the aftereffect to last longer; changes persisting for more than one hour were reported after prolonged adaptation (Gibson and Radner, 1937; Blakemore et al., 1970; Heggelund and Hohmann, 1976; Mecacci and Spinelli, 1976). However, Mecacci and Spinelli (1976) reported that the amplitude reduction observed in the human visual evoked potential first stabilized after 15 min adaptation to high-contrast gratings. Some later psychophysical results by Bodinger (1978) likewise suggested that it might take longer adaptation times to reach magnitude saturation, and recent extensive experiments on the threshold elevation and tilt aftereffects show that there is a steady growth during at least 20-30 min of adaptation (Björklund and Magnussen, 1981; Rose and Evans, 1983; Magnussen and Johnsen, 1986). There is no evidence for saturation in these experiments.

*Present address: Institute of Psychology, University of Oslo, Box 1094, Blindern, N-0317 Oslo 3, Norway.
Neurophysiological studies of adaptation in single cells of the cat's visual cortex have mostly used shorter adaptation durations leading to comparatively short-lived aftereffects (Maffei et al, 1973; Vautin and Berkley, 1977; Movshon and Lennie, 1979; Dean, 1983). However, Creutzfeldt and Heggelund (1975) exposed adult cats to high-contrast vertical gratings for two one-hour sessions per day over a period of 2 weeks. Between exposures the cats were kept in darkness. When later tested for cortical organization, these cats were found to possess very few responsive cells whose preferred orientation matched that of the adapting gratings. These results, in conjunction with the psychophysical studies mentioned above, suggest that spatial adaptation phenomena might be more profound than hitherto realized. We therefore decided to test the limits of spatial adaptation by tracking the changes in grating contrast thresholds during a marathon session of adaptation.

\section{METHOD}

The spatial dimensions of the adapting and test fields are shown in the inset to Fig. 1. Since it is difficult to attend for several hours to a spatially restricted stimulus containing a small fixation line or circle, the adapting field was much larger than the test field, making accurate fixation less critical. The adapting grating subtended $11 \times 16 \mathrm{deg}$, the test grating $4 \times 5 \mathrm{deg}$ visual angle. Not shown in the figure are a $0.75 \mathrm{deg}$ fixation circle in the center of the adapting grating and a fixation point in the center of the test grating. The adapting grating was produced by back-projection on a translucent screen, a test grating of variable contrast was generated on a Tektronix 602 CRT using a Picasso image generator (Innisfree Ltd). The spatial frequency of the adapting 
and test gratings was $6.0 \mathrm{c} / \mathrm{deg}$, the mean luminance was $30 \mathrm{~cd} / \mathrm{m}^{2}$, and the contrast of the adapting grating was 0.6 . Contrast is defined as the difference between the grating's maximum and minimum luminances divided by their sum. The test grating was turned on and off at a frequency of $0.5 \mathrm{~Hz}$ (squarewave time modulation).

Test-grating contrast was controlled via a 10-turn logarithmic potentiometer operated by the experimenter. Contrast thresholds were measured by a modified method of adjustment. Starting from an arbitrary subthreshold value, contrast was gradually increased until the grating was reported as being just visible. The duration of the test periods was $5 \mathrm{sec}$. Exposures of the test grating alternated with either $15 \mathrm{sec}$ exposures of a blank field of equal mean luminance (when measuring pre-adaptation and decay thresholds) or with 15-sec readaptation periods. Beginning and end of the test periods were marked by an auditory signal. The adapting field was placed immediately above the test field, and the subject shifted his gaze contingent on the signal.

The subject was comfortably seated, resting on a chin- and forehead support. During adaptation he moved his eyes along the edges of the fixation circle, but was allowed occasional excursions to other parts of the adapting field. Though proving unnecessary, we were even prepared to allow short breaks; we have evidence that interposed blank intervals of less than $60 \mathrm{sec}$ duration, if anything, enhances the adaptation effect (Magnussen and Greenlee, 1985).

Contrast thresholds were measured at regular 10-30 min intervals during adaptation. Each thres- hold determination consisted of ten settings made on a test-readapt-test schedule. Adaptation was terminated when similar values turned up in at least four successive threshold measurements. Thus adaptation was continued for at least two hours after the saturation point was attained. The subject was given no feedback during the experiment, and the second subject run was ignorant about the previous data.

During recovery, threshold settings were made as quickly as possible during the first few minutes. Thereafter measurements were made at regular intervals and the subject was free to move around in between. The experiment was terminated when the contrast thresholds has returned to a value of $2 \mathrm{SE}$ above the pre-adaptation baseline values. Measurements made on several occasions prior to the main experimental session indicated a very stable baseline; the values reported in Fig. 1 are the means of 20 settings made immediately prior to adaptation.

Subjects were author S.M. and a paid naive subject R.H. familiar with spatial adaptation experiments.

\section{RESULTS}

Fortunately, the day- and night-session we had prepared for proved unnecessary as a plateau was reached earlier than expected. Figure l(a) plots on double logarithmic axes the contrast needed to detect the test grating as a function of adaptation time. To permit a more precise location of the early data points on the time axis, the thresholds for adaptation times shorter than $10 \mathrm{~min}$ were determined in separate experiments. The results show two-branched (a)

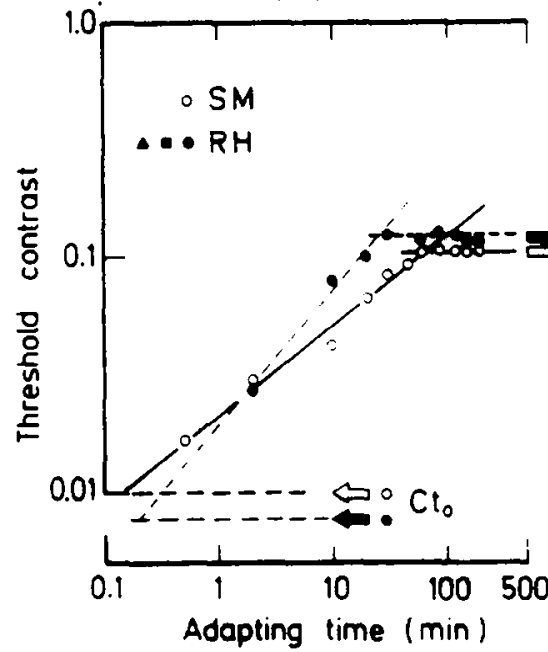

(b)

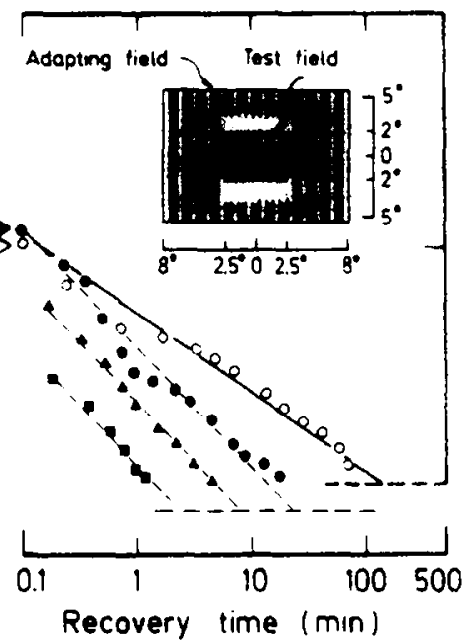

Fig. 1(a) The contrast required to detect a $6.0 \mathrm{c} / \mathrm{deg}$ vertical grating as a function of time adapting to a high-contrast (0.6) grating of the same spetial frequency and orientation. Dashed lines and arrows indicate the unadapted (baseline, $\left.C t_{0}\right)$ thresholds of the two subjects tested $(n=20)$. (b) Time-course of the recovery to normal sensitivity following adaptation. Results from the marathon adaptation are shown by solid and open circles; $n=10$, except during the carly decay phase where only 1 or 2 settings define a threshold. Solid squares and triangles show the decay following 2 and $10 \mathrm{~min}$ adaptation measured for R.H.: average results for three runs. Regression lines are fitted by least-squares method. Inset shows a scaled-down representation of the relative sizes of the adapting and test gratings. In the experiments the adapting grating was located directly above the test grating. 
growth curves, each branch was fitted by a regression line. The horizontal segment defines the saturation level, and the intercept between regression lines the saturation time. There are individual differences to be noted: first, in baseline contrast sensitivity $\left(C t_{0}\right)$, indicated by horizontal dashed lines; second, in the magnitude of the threshold elevation at saturation level (approx, $1.0 \mathrm{log}$ unit for S.M. and $1.2 \mathrm{log}$ units for R.H.); and third, S.M. took more time to reach saturation than R.H. (approx. $1 \mathrm{hr}$ vs $1 / 2 \mathrm{hr}$ ). Otherwise, the results are very similar for the two subjects.

Adaptation was terminated after three hours. The time course of the subsequent decay is shown for the two subjects in Fig. 1(b) as solid and open circles. The return to normal sensitivity was much quicker for subject R.H. than for S.M., a difference which mirrors the intersubject difference in growth rate.

In separate experiments decay functions following 2 and 10 min adaptation were measured for subject R.H. These results are shown in Fig. 1(b) by squares and triangles, respectively. Note that these decay functions have the same slope as the decay from the 3-hr adaptation.

\section{DISCUSSION}

Prolonged inspection of a high-contrast grating leads to a decline in contrast sensitivity for test gratings of similar spatial frequencies and orientations. This decline progresses over a much longer time than indicated by the early experiments (Blakemore and Campbell, 1969). For the two subjects tested in the present experiments saturation was reached after 30 and $60 \mathrm{~min}$ of adaptation, respectively, at which point the contrast threshold had increased $1.0-1.2 \mathrm{log}$ units out of a range of less than $2 \mathrm{log}$ units, assuming that the contrast needed to detect the test grating following adaptation cannot exceed the adapting contrast itself, in casu 0.6. Increasing the adapting contrast might push the limits of spatial adaptation somewhat further, but it is unlikely that the change would be large.

A shorter saturation time is associated with a quicker recovery. The individual growth and decay functions of the aftereffect have approximately the same slope, thus adaptation and recovery appear to be fairly symmetric processes. This has also been reported for the tilt aftereffect (Magnussen and Johnsen, 1986)

Data reported by Björklund and Magnussen (1981) indicate that the decay functions have a constant slope across adaptation times when plotted on $\log -\log$ axes. The results of Fig. 1(b) confirm and extend this invariance to very long adaptation times. This suggests that adaptation beyond the saturation point has no further influence on the subsequent rate of recovery. Furthermore, we have no evidence for any residual long-term baseline threshold change as was reported by Wolfe (1984) for the tilt aftereffect.

Acknowledgements-SM thanks the Alexander von Humboldt Foundation (Bonn, F.R.G.) and the Norwegian Research Council for Science and the Humanities for support during a sabbatical leave in Freiburg. M.W.G. was supported by the Deutsche Forschungsgemeinschaft (SFB 70 , A6; to L. Spillmann). We thank L. Spillmann for providing laboratory facilities and $\mathbf{R}$. Hessenkamp for serving as a subject.

\section{REFERENCES}

Björklund R. A. and Magnussen S. (1981) A study of the interocular transfer of spatial adaptation. Perception 10, $511-518$.

Blakemore C. and Campbell F. W. (1969) On the existence of neurones in the human visual system selectively sensitive to the orientation and size of retinal images. J. Physiol. 203, 237-260.

Blakemore C., Muncey J. P. J. and Ridley R. M. (1973) Stimulus specificity in the human visual system. Vision Res. 13, 1915-1931.

Blakemore C., Nachmias J. and Sutton P. (1970) The perceived spatial frequency shift: evidence for frequency selective neurones in the human brain. $J$. Physiol. 210 , $727-750$

Bodinger D. M. (1978) The decay of grating adaptation. Vision Res. 28, 89-91.

Braddick O., Campbell F. W. and Atkinson J. (1978) Channels in vision: Basic aspects. In Handbook of Sensory Physiology (Edited by Held R., Leibowitz H. W. and Teuber H.-L.), Vol. VIII, pp. 3-38. Springer, Berlin.

Creutzfeld $O$. D. and Heggelund P. (1975) Neural plasticity in the visual cortex of adult cats after exposure to visual patterns. Science 188, 1025-1027.

Dean D. F. (1983) Adaptation-induced alteration of the relation between response amplitude and contrast in cat striate cortical neurones. Vision Res. 23, 249-256.

Gibson J. J. and Radner M. (1937) Adaptation, aftereffect and contrast in the perception of tilted lines: I. Quantitative studies. J. exp. Psychol. 20, 453-467.

Heggelund P. and Hohmann A. (1976) Long-term retention of the "Gilinsky-effect", Vision Res. 16, 1015-1017.

Mecacei L. and Spinelli D. (1976) The effects of spatial frequency adaptation on human evoked potentials. Vision Res: 16, 477-479.

Maffei L., Fiorentini A. and Bisti S. (1973) Neural correlate of perceptual adaptation to gratings. Science 182 1036-1038.

Magnussen S. and Greenlee M. W. (1985) Contrast threshold elevation following continuous and interrupted adaptation. In preparation.

Magnussen S. and Johnsen T. (1986) Temporal aspects of spatial adaptation. A study of the tilt aftereffect. To be published.

Movshon J. A. and Lennie P. (1979) Pattern-selective adaptation in visual cortical neurones. Nature 278 , $850-852$

Rose D. and Evans R. (1983) Evidence against saturation of contrast adaptation in the human visual system. Percept. Psychophys. 34, 158-160.

Vautin R. G. and Berkley M. A. Responses of single cells in cat visual cortex to prolonged stimulus movement. Neural correlates of visual aftereffects. $J$. Neurophysiol. 40, 1051-1065.

Wolfe J. M. (1984) A constructive model of visual aftereffects. Perception 13, Al4 